\title{
Influencia del curado sobre la evolución de la corrosión en vigas de hormigón armado con diferentes agregados finos
}

\author{
O. A. Cabrera ${ }^{1}$, N. F. Ortega ${ }^{2}$, M. I. Schierloh ${ }^{3}$, L. P. Traversa ${ }^{4}$
}

${ }^{1}$ Facultad de Ingeniería, U.N.C.P.B.A., Av. del Valle 5737, (7400) - Olavarría, Argentina.

${ }^{2}$ Departamento de Ingeniería, Universidad Nacional del Sur, Av. Alem 1253, (8000) - Bahía Blanca, Argentina.

${ }^{3}$ U.T.N., F.R. Concepción del Uruguay, Ing. Pereira 676, (3264) - Concepción del Uruguay, Argentina.

${ }^{4}$ Laboratorio de Entrenamiento Multidisciplinario para la Investigación Tecnológica (CIC-LEMIT), Calles 52 y 121, (1900) La Plata, Argentina.

\section{Información del artículo DOI: \\ http://dx.doi.org/10.21041/ra.v $\underline{2 i 2.28}$ \\ Artículo recibido el 13 de Febrero de 2012, revisado bajo las políticas de publicación de la Revista ALCONPAT y aceptado el 23 de Marzo de 2012. Cualquier discusión, incluyendo la réplica de los autores se publicará en el primer número del año 2013 siempre y cuando la información se reciba antes del cierre del tercer número del año 2012}

(C) 2012 ALCONPAT Internacional

Información Legal

Revista ALCONPAT, Año 2, No. 2, Mayo Agosto 2012, es una publicación cuatrimestral de la Asociación Latinoamericana de Control de Calidad, Patología y Recuperación de la Construcción, Internacional, A.C., Km. 6, antigua carretera a Progreso, Mérida Yucatán, C.P. 97310, Tel.5219997385893, alconpat.int@gmail.com, Página Web: www.alconpat.org Editor responsable: Dr. Pedro Castro Borges. Reserva de derechos al uso exclusivo No.042013-011717330300-203, eISSN 2007-6835, ambos otorgados por el Instituto Nacional d Derecho de Autor. Responsable de la última actualización de este número, Unidad de Informática ALCONPAT, Ing. Elizabeth Sabido Maldonado, Km. 6, antigua carretera a Progreso, Mérida Yucatán, C.P. 97310 , fecha de publicación: 30 de Mayo de 2012.

Las opiniones expresadas por los autores no necesariamente reflejan la postura del editor. Queda totalmente prohibida la reproducción total o parcial de los contenidos e imágenes de la publicación sin previa autorización de la ALCONPAT Internacional A.C.

\section{RESUMEN}

En este trabajo se analiza cómo el tipo de curado afecta la microestructura de hormigones con diferentes agregados finos, respecto al ingreso de fluidos, que se vinculan estrechamente con el deterioro del acero. Se evaluaron de 5 grupos distintos de probetas y vigas de hormigón armado, con curados: normal, en ambiente de laboratorio, en mufla, en ambiente frío y bajo la acción del viento. Las vigas fueron sometidas a ensayos de corrosión acelerada, en presencia de cloruros. Se concluyó que la velocidad de succión capilar es muy sensible al tipo de curado. En la corrosión de las vigas, el curado defectuoso influyo fuertemente sobre el tiempo de aparición de la primera fisura, el área y el ancho máximo de fisuras.

Palabras claves: arena triturada; forma y textura superficial; hormigón armado; corrosión, cloruros.

\section{ABSTRACT}

This paper analyses how the type of curing affects concrete microstructures with different fine aggregates in relation to the ingress of fluids closely connected with steel deterioration. Five different groups of specimens and beams of reinforced concrete were evaluated with curing in normal conditions, at laboratory ambient, in muffle furnace, at cold ambient and under wind action. Beams were submitted to accelerated corrosion tests in the presence of chlorides. It was concluded that capillary suction is very sensitive to the type of curing. In beam corrosion, defective curing highly influenced the time of appearance of the first crack, the area and maximum wide of cracks.

Keywords: crushed sand; shape and surface texture; reinforced concrete; corrosion; chlorides.

Autor de contacto: O. A. Cabrera (ocabrera@fio.unicen.edu.ar) 
Revista ALCONPAT, Volumen 2, Número 2, Mayo - Agosto 2012, Páginas 72 - 81

\section{INTRODUCCIÓN}

La corrosión de las armaduras es una de las principales patologías de las estructuras de hormigón armado, que implica una merma de la seguridad estructural, la disminución de la vida útil y el uso poco racional de los recursos no renovables que determina que las estructuras no sean sustentables (Alonso et al., 1998; Traversa, 2001). El hormigón es el encargado de proteger a las armaduras de la acción de los agentes externos, por constituir una barrera física y también, por brindar un medio alcalino en el cual el acero permanece pasivo (DURAR, 2000).

La capilaridad es uno de los mecanismos de transporte que tiene una importancia significativa en los procesos de deterioro, siendo para los fluidos el mecanismo de ingreso más rápido, y la determinación de la succión capilar permite caracterizar de modo comparativo la estructura de poros. De esta manera, la velocidad de succión capilar del hormigón y, particularmente del recubrimiento, están asociadas con la durabilidad de las estructuras. El método es sensible a los cambios en las mezclas y, particularmente, a las condiciones de curado, resultando una herramienta eficaz para especificaciones basadas en el desempeño del material (Cabrera et al., 2006). En hormigones convencionales, los mecanismos de transporte se producen por los capilares de la pasta de cemento y la interfaz "pasta-agregado", siendo esta última una zona de alta porosidad, facilitando la penetración de sustancias agresivas para las armaduras (cloruros, $\mathrm{O}_{2}, \mathrm{CO}_{2}$, etc.). Además, la forma, textura superficial y tamaño de los granos de los agregados finos inciden sobre la trabajabilidad de las mezclas, por cuanto la fricción entre partículas y la superficie específica pueden incrementar el contenido de pasta; y entonces, afectar a la resistencia y la durabilidad de los hormigones (Cabrera et al., 2011).

Por otra parte, el curado influye significativamente sobre las propiedades del hormigón endurecido, tales como su resistencia mecánica, permeabilidad, resistencia a la abrasión, estabilidad de volumen y resistencia a los ciclos de congelación y deshielo, etc. El desarrollo de la resistencia superficial puede reducirse cuando el curado es defectuoso, porque la evolución de la hidratación se ve retrasada y/o interrumpida, lo que provoca una menor cantidad de productos de hidratación, quedando una parte del cemento anhidro.

La consecuencia de un curado defectuoso que afecta la microestructura del hormigón, se manifiesta en que la porosidad capilar y la conectividad de poros son mayores que en un curado normal. Este cambio en la hidratación lleva a la reducción de la resistencia y al incremento de la permeabilidad del hormigón del recubrimiento. En resumen, el comportamiento durable del hormigón armado depende, en gran medida, del diseño de la mezcla, la selección de los materiales, del control en la colocación, compactación y de las condiciones de curado. En el presente trabajo experimental se analiza como influye el tipo de agregado fino y el modo de curado, sobre el proceso de corrosión de las armaduras.

\section{MATERIALES, DOSIFICACIONES Y METODOLOGÍA}

Hormigón y acero: en este estudio se analizan los resultados obtenidos sobre vigas de hormigón armado sometidas a un proceso de corrosión acelerado. Para ello se diseñaron 4 series de hormigones, con 3 arenas naturales de idéntico origen geológico y una arena triturada granítica, con diferentes módulos de finura, 2.30, 2.54, 2.80 y 2.88, respectivamente (Tabla 1). Como agregado grueso se usó una piedra partida cuarcítica, con un tamaño máximo nominal de 13.5 $\mathrm{mm}$, y el aglomerante fue un cemento Portland normal CPN 40 (IRAM 50000, 2000). Los hormigones fueron elaborados con $300 \mathrm{~kg} / \mathrm{m}^{3}$ de cemento y una relación agua/cemento fue de 0.60, manteniendo constante el volumen de pasta de cemento. Las mezclas se denominan con la letra $\boldsymbol{M}$ y el correspondiente número de arena. Los ensayos realizados sobre el hormigón endurecido fueron: resistencia a compresión (IRAM 1546, 1992), y la determinación de la 
Revista ALCONPAT, Volumen 2, Número 2, Mayo - Agosto 2012, Páginas 72 - 81

velocidad de succión capilar a las 24 hs (VSC). La resistencia a rotura del acero de dureza natural de las barras longitudinales fue de $855 \mathrm{MPa}$.

Tabla 1. Características de los agregados finos.

\begin{tabular}{|l|c|c|c|c|}
\hline Característica & Fina Nat. (1) & Triturada (2) & Gruesa Nat. (3) & Mezcla Nat. (4) \\
\hline Módulo de finura & 2.30 & 2.88 & 2.80 & 2.54 \\
\hline Contenido de polvo $(<75 \mu \mathrm{m})$ & $1 \%$ & $4 \%$ & $1 \%$ & $1 \%$ \\
\hline Densidad relativa & 2.58 & 2.63 & 2.58 & 2.58 \\
\hline Tipo de agregado & Silíceo & Granítico & Silíceo & Silíceo \\
\hline
\end{tabular}

Vigas de hormigón armado: las vigas de 110 × 10 × $15 \mathrm{~cm}$ se construyeron con 4 barras de acero de $4.2 \mathrm{~mm}$ de diámetro y con estribos de $2.1 \mathrm{~mm}$ de diámetro, separados cada $10 \mathrm{~cm}$. El espesor del recubrimiento de las barras fue de $10 \mathrm{~mm}$. Las vigas y probetas fueron curadas de 5 modos diferentes que se indican a continuación:

Tipos de curado: se modelaron distintas situaciones relacionadas con el curado que suelen presentarse en la práctica habitual de la construcción, que distan del curado ideal o normal. Las probetas y vigas se dividieron en 5 grupos que fueron curados de la siguiente manera: normal $(C N$ : a $20 \pm 2{ }^{\circ} \mathrm{C}$ y HR $>95 \%$ durante 28 días), en ambiente de laboratorio (CL: a $20 \pm 2{ }^{\circ} \mathrm{C}$ y $\mathrm{HR} \cong 50$ $\%$ hasta completar 28 días), en mufla (CM: a $40 \pm 5^{\circ} \mathrm{C}$ y $\mathrm{HR} \cong 13 \%$ durante los primeros 7 días, y luego en ambiente de laboratorio hasta los 28 días), en ambiente exterior ( $C E$ : estacionadas en época de invierno a una temperatura media de $6{ }^{\circ} \mathrm{C}$ durante 28 días) y bajo la acción del viento ( $C L V$ : simulando en laboratorio con una velocidad de $10 \pm 1 \mathrm{Km} / \mathrm{h}$, aplicado durante los primeros 7 días, y luego se mantuvieron en laboratorio hasta completar 28 días).

Proceso de corrosión acelerada: en este ensayo se aplicó una corriente de $100 \mu \mathrm{A} / \mathrm{cm}^{2}$ provista por un galvanostato sobre la superficie del hormigón, en la parte inferior de la viga en el sentido de llenado, a través de un contraelectrodo formado por una malla de acero (de $50 \mathrm{~cm}$ de largo). Sobre el mismo se dispuso una esponja para humectar con una solución de cloruros de sodio al $0.3 \%$ en peso. Esta metodología se ha empleado satisfactoriamente en trabajos anteriores (Ortega y Aveldaño, 2008; Aveldaño y Ortega, 2009; Rodríguez et al., 1993; Schierloh, 2002).

A partir del surgimiento de las fisuras, se efectuó periódicamente la medición del largo y ancho de las mismas, para luego calcular las áreas de fisuración. Además, se registraron los potenciales de corrosión con un electrodo de referencia de $\mathrm{Cu} / \mathrm{CuSO}_{4}$ (ASTM C-876, 2000), luego se descubrieron las barras, relevándose su deterioro y, finalmente, se realizó una gravimetría, para determinar las particularidades de la corrosión registrada en cada caso.

\section{ANÁLISIS Y DISCUSIÓN DE LOS RESULTADOS}

\subsection{Evaluación del hormigón}

En la Tabla 2 se muestran los valores correspondientes a los principales parámetros del estado endurecido que caracterizan a los hormigones en estudio, elaborados con distintos tipos de agregados finos y expuestos a diferentes tipos de curados. En base a estos resultados, a continuación se efectúa el correspondiente análisis del comportamiento de dichos hormigones. 
Revista ALCONPAT, Volumen 2, Número 2, Mayo - Agosto 2012, Páginas 72 - 81

Tabla 2. Propiedades de los hormigones con diferentes tipos de curados.

\begin{tabular}{|c|c|c|c|c|c|c|c|c|}
\hline \multirow{2}{*}{ Propiedades } & \multicolumn{4}{|c|}{ Curado normal } & \multicolumn{4}{|c|}{ Curado exterior } \\
\hline & $M-1$ & $M-2$ & $M-3$ & $M-4$ & $M-1$ & $M-2$ & $M-3$ & $M-4$ \\
\hline Resistencia a 28 días (MPa) & 33 & 38 & 25 & 32 & 18.8 & 21.3 & 21.5 & 20.9 \\
\hline$V S C$ a $24 \mathrm{~h}\left(\mathrm{~g} / \mathrm{m}^{2} . \mathrm{s}^{0.50}\right)$ & 2.89 & 20.9 & 17.6 & 6.7 & 20.2 & 25.62 & 26.41 & 25.43 \\
\hline Propiedades & \multicolumn{4}{|c|}{ Curado en laboratorio } & \multicolumn{4}{|c|}{ Curado en mufla } \\
\hline Resistencia a 28 días (MPa) & 24 & 23 & 18 & 24 & 17 & 14 & 14 & 19 \\
\hline$V S C$ a $24 \mathrm{~h}\left(\mathrm{~g} / \mathrm{m}^{2} \cdot \mathrm{s}^{0.50}\right)$ & 27.0 & 36.7 & 38.8 & 21.2 & 30.8 & 23.1 & 35.4 & 35.6 \\
\hline Propiedades & \multicolumn{4}{|c|}{ En laboratorio con viento } & & & & \\
\hline Resistencia a 28 días (MPa) & 16.5 & 23.9 & 17.1 & 19.0 & & & & \\
\hline$V S C$ a $24 \mathrm{~h}\left(\mathrm{~g} / \mathrm{m}^{2} \cdot \mathrm{s}^{0.50}\right)$ & 24.07 & 24.12 & 29.51 & 25.99 & & & & \\
\hline
\end{tabular}

Resistencia a compresión: la resistencia de los hormigones con $C N$, a los 28 días, fue de $32 \pm 7$ $\mathrm{MPa}$. La resistencia de la mezcla $M-2$, con arena triturada, resultó un $29 \%$ mayor que el promedio de las resistencias con arenas naturales. Aquellos con curados $C L$ y $C M$ presentaron una resistencia de $21 \pm 3,5 \mathrm{MPa}$ y $16 \pm 3 \mathrm{MPa}$, respectivamente. Es decir, que existió una disminución en la resistencia del 25 al $41 \%$ y del 40 al $62 \%$, respecto a los valores correspondientes al $C N$. Los hormigones curados $C E$ y en $C L V$ se mantuvieron en un rango intermedio entre los $C M$ y $C L$. Velocidad de succión capilar: la VSC de los hormigones, a los 28 días con $C N$, fue de $12 \pm 9$ $\mathrm{g} / \mathrm{m}^{2} \cdot \mathrm{s}^{0.50}$. Los hormigones con curados $C L$ y $C M$ presentaron una velocidad mayor de $30 \pm 12$ $\mathrm{g} / \mathrm{m}^{2} . \mathrm{s}^{0.50}$ y $30 \pm 10 \mathrm{~g} / \mathrm{m}^{2} . \mathrm{s}^{0.50}$, respectivamente. El incremento en los valores con respecto a los del $C N$ fue del 180 al $930 \%$ y del 110 al $1070 \%$, respectivamente. Los hormigones con curados $C E$ y $C L V$ presentan un comportamiento similar a los $C L$ y $C M$.

Los parámetros analizados que caracterizan al hormigón endurecido muestran que la VSC presenta una gran sensibilidad respecto al tipo de curado. En cambio, los efectos sobre la resistencia a compresión del tipo de curado son menores en varios órdenes de magnitud. Esto se puede asociar a que la VSC evalúa fenómenos que afectan la parte exterior de la probeta, en cambio la resistencia es el resultado de la calidad de todo el volumen de hormigón.

\subsection{Comportamiento de vigas de hormigón armado sometidas a corrosión acelerada}

Seguimiento de la fisuración: una forma de analizar el progreso del daño ocasionado en las vigas por la corrosión de las armaduras, es midiendo las áreas de fisuras (definida como la sumatoria del ancho por el largo de fisura de cada tramo de dicha anchura) de un modo acumulativo. La formación de óxidos provoca el crecimiento de las fisuras, tanto en ancho como en largo. El ancho máximo de la fisura no siempre se va incrementando en la misma zona, sino que va variando de sector fisurado. En la corrosión del acero se expresa la pérdida teórica de masa ocurrida como la pérdida radial de la sección de la barra, que se denomina "profundidad media de penetración de la corrosión, PM" (Torres-Acosta y Sagües, 1998), calculada en base a la ley de Faraday.

Las Figuras 1 a 5 muestran la evolución de la fisuración frente al incremento de la profundidad teórica para las vigas con los curados $C N, C E, C L, C L V$ y $C M$, respectivamente. Los valores del área acumuladas de fisuras para las 4 mezclas resultan mayores en el caso del $C N$, respecto a los otros curados. Este hecho se asocia a la menor porosidad del hormigón con $C N$, que permite que los productos de corrosión ocupen los poros completamente, y de este modo, se incremente la presión interna originada por el mayor volumen de los óxidos, respecto al volumen de metal corroído, originando una mayor fisuración o grado de deterioro de las vigas. 
Revista ALCONPAT, Volumen 2, Número 2, Mayo - Agosto 2012, Páginas 72 - 81

Al final del ensayo, la relación entre el daño mayor y el menor de las vigas para el grupo con $C N$ es del $86 \%$ (Figura 1), y de $367 \%$ y $343 \%$, para los grupos con curados $C L$ y $C M$, respectivamente. Si bien los valores del daño de las vigas, medidos con las áreas de fisuras, son mayores en el caso del $C N$, los curados $C L$ y $C M$ muestran una mayor dispersión para las distintas mezclas. La menor dispersión se obtuvo para los curados $C L V$ y $C E, 47 \%$ y $52 \%$, respectivamente.

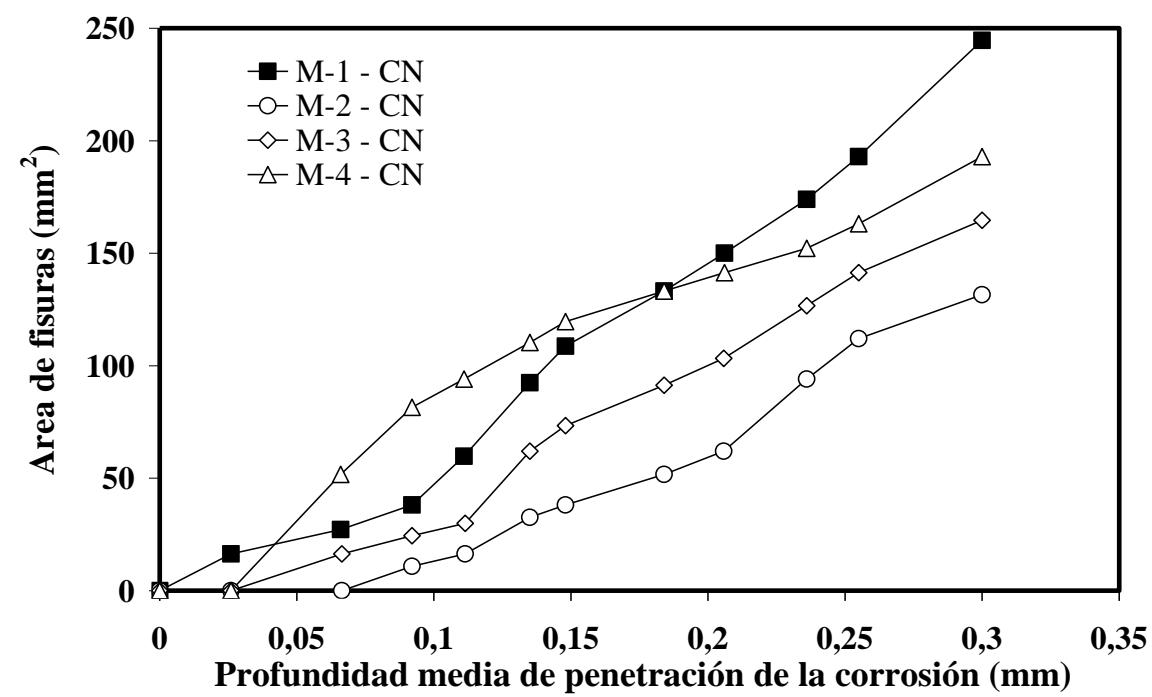

Figura 1. Evolución de la fisuración con la $P M$ para hormigones con curado $C N$.

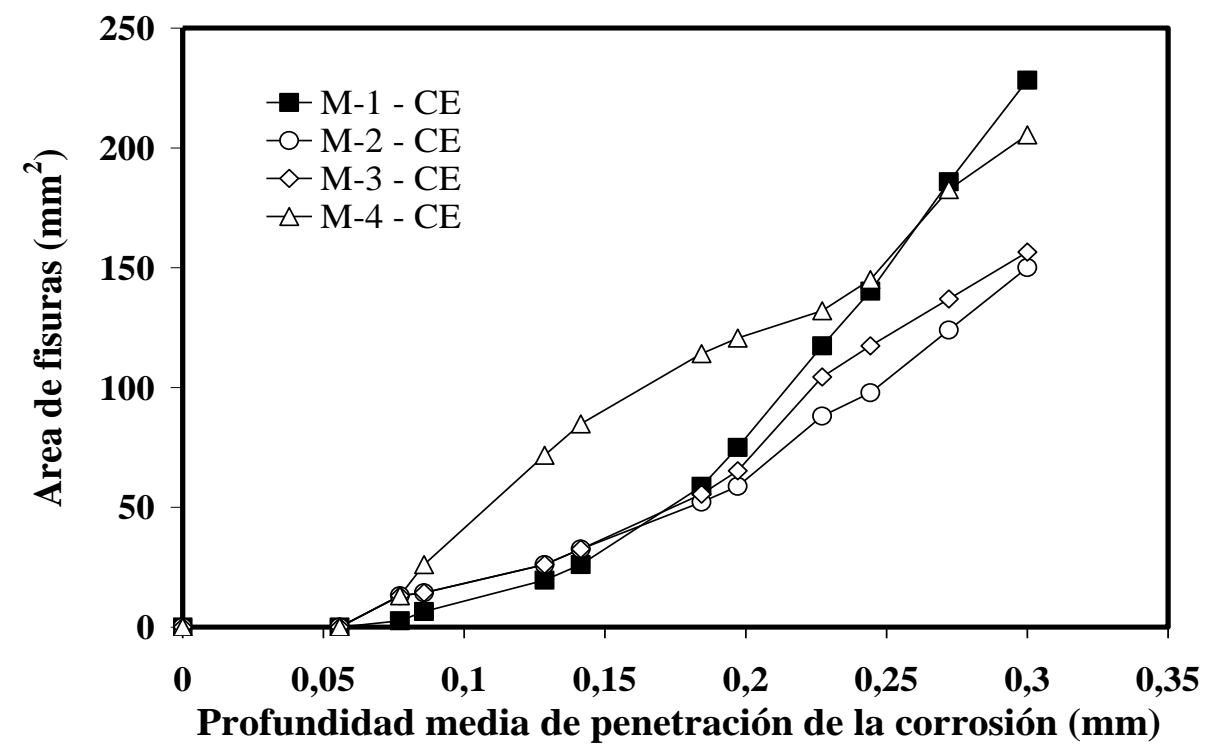

Figura 2. Evolución de la fisuración con la $P M$ para hormigones con curado $C E$. 
Revista ALCONPAT, Volumen 2, Número 2, Mayo - Agosto 2012, Páginas 72 - 81

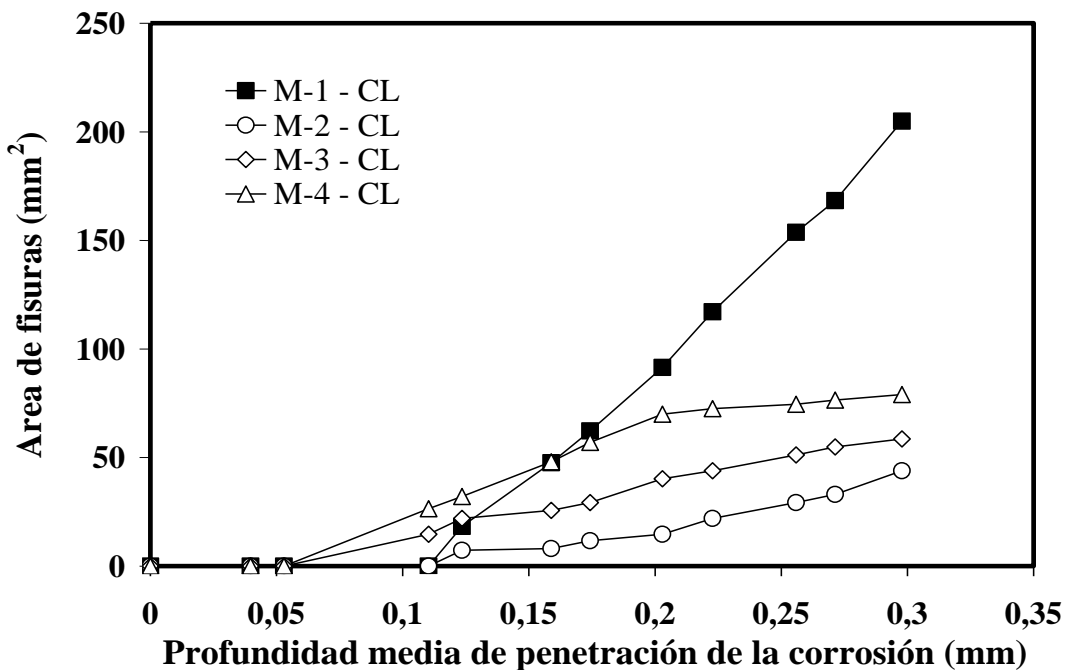

Figura 3. Evolución de la fisuración con la $P M$ para hormigones con curado $C L$.

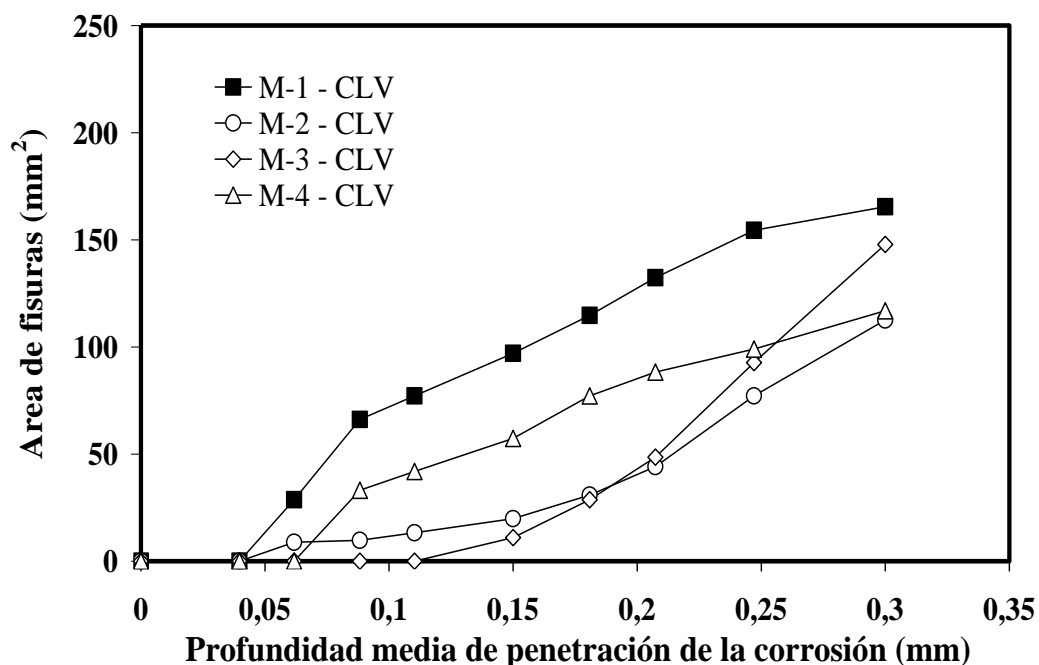

Figura 4. Evolución de la fisuración con la $P M$ para hormigones con curado $C L V$.

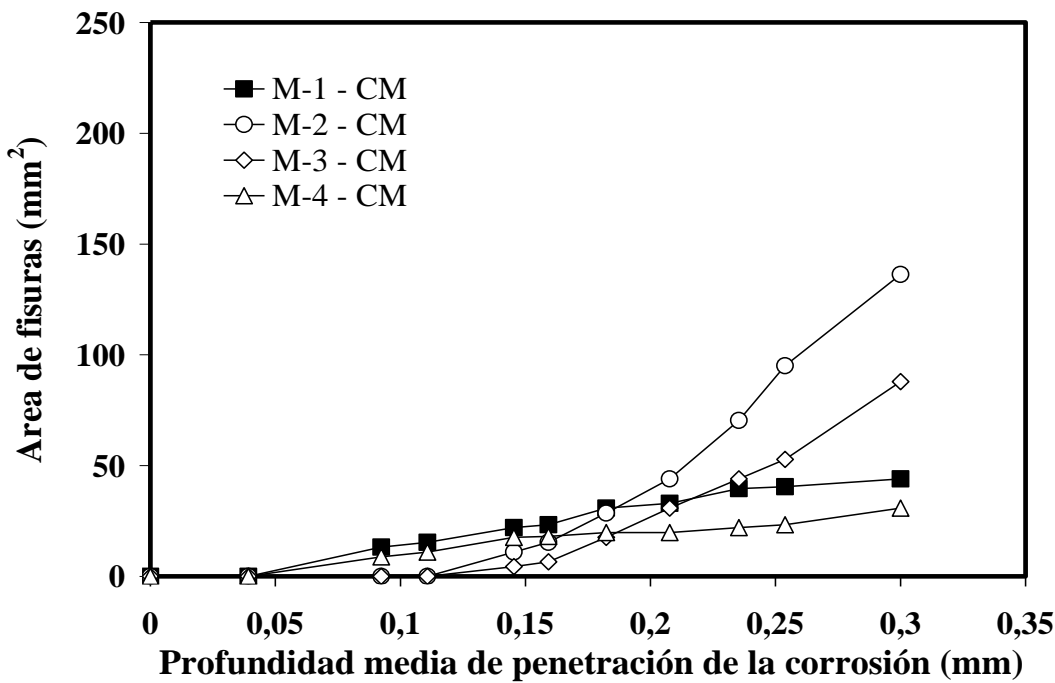

Figura 5. Evolución de la fisuración con la $P M$ para hormigones con curado $C M$. 
Revista ALCONPAT, Volumen 2, Número 2, Mayo - Agosto 2012, Páginas 72 - 81

Ajustando la variación mediante una regresión lineal a las curvas de las Figura 1 a 5, en el sector que abarca la mayor parte del tiempo de ensayo (hasta los 110 días), se obtuvo un gráfico similar al del modelo de Tutti ${ }^{3}$ que vincula al daño de las vigas con el $P M$, que varía linealmente con el tiempo (período de propagación). De este modo, en todos los casos se obtuvieron las ecuaciones que vinculan el área de fisuración $\left(A F\right.$, en $\left.\mathrm{mm}^{2}\right)$ con la $P M(\mathrm{en} \mathrm{mm})$ con adecuados coeficientes de correlación, que se indican en la Tabla 3,

Tabla 3. Relación entre el daño de las vigas y la profundidad media de penetración de la corrosión, en el periodo de propagación; y tiempo teórico de aparición de la primera fisura obtenidos de las

Figuras 1 a 5.

\begin{tabular}{|c|c|c|c|c|}
\hline Mezcla & Curado & $\begin{array}{l}\text { Área en función de la } \boldsymbol{P M} \text {, } \\
\left(\mathrm{AF} \text { en } \mathrm{mm}^{2} \text {, y } \mathrm{PM} \text { en } \mathrm{mm}\right)\end{array}$ & $\mathbf{R}^{2}$ & $\begin{array}{l}\text { Tiempo de aparición de } \\
\text { la primera fisura (días) }\end{array}$ \\
\hline$M-1$ & \multirow{4}{*}{$C N$} & $\mathrm{AF}=866.7 * \mathrm{PM}-26.14$ & 0.98 & $8(100 \%)$ \\
\hline$M-2$ & & $\mathrm{AF}=485.3 * \mathrm{PM}-24.92$ & 0.94 & $21(100 \%)$ \\
\hline$M-3$ & & $\mathrm{AF}=631.8 * \mathrm{PM}-25.37$ & 0.99 & $21(100 \%)$ \\
\hline$M-4$ & & $\mathrm{AF}=623.3 * \mathrm{PM}-13.03$ & 0.94 & $8(100 \%)$ \\
\hline$M-1$ & \multirow{4}{*}{$C L$} & $\mathrm{AF}=1069.3 * \mathrm{PM}-120.02$ & 0.99 & $28(350 \%)$ \\
\hline$M-2$ & & $\mathrm{AF}=211.5 * \mathrm{PM}-23.84$ & 0.95 & $35(170 \%)$ \\
\hline$M-3$ & & $\mathrm{AF}=222.78 * \mathrm{PM}-7.11$ & 0.98 & $27(130 \%)$ \\
\hline$M-4$ & & $\mathrm{AF}=294.43 * \mathrm{PM}-2.65$ & 0.91 & $17(210 \%)$ \\
\hline$M-1$ & \multirow{4}{*}{$C M$} & $\mathrm{AF}=173.5 * \mathrm{PM}-3.79$ & 0.98 & $15(190 \%)$ \\
\hline$M-2$ & & $\mathrm{AF}=793.5 * \mathrm{PM}-106.73$ & 0.97 & $35(170 \%)$ \\
\hline$M-3$ & & $\mathrm{AF}=588.5 * \mathrm{PM}-88.79$ & 0.98 & $35(170 \%)$ \\
\hline$M-4$ & & $\mathrm{AF}=104.6 * \mathrm{PM}-0.96$ & 0.94 & $15(190 \%)$ \\
\hline$M-1$ & \multirow{4}{*}{$C E$} & $\mathrm{AF}=785.6 * \mathrm{PM}-53.59$ & 0.86 & $21(260 \%)$ \\
\hline$M-2$ & & $\mathrm{AF}=513.6 * \mathrm{PM}-27.18$ & 0.92 & $17(80 \%)$ \\
\hline$M-3$ & & $\mathrm{AF}=570.7 * \mathrm{PM}-30.84$ & 0.92 & $17(80 \%)$ \\
\hline$M-4$ & & $\mathrm{AF}=740.8 * \mathrm{PM}-27.11$ & 0.97 & $12(150 \%)$ \\
\hline$M-1$ & \multirow{4}{*}{$C L V$} & $\mathrm{AF}=557.7 * \mathrm{PM}+10.70$ & 0.96 & $6(75 \%)$ \\
\hline$M-2$ & & $\mathrm{AF}=357.7 * \mathrm{PM}-17.92$ & 0.86 & $17(80 \%)$ \\
\hline$M-3$ & & $\mathrm{AF}=466.8 * \mathrm{PM}-31.80$ & 0.79 & $21(100 \%)$ \\
\hline$M-4$ & & $\mathrm{AF}=403.0 * \mathrm{PM}-0.81$ & 0.99 & $1(13 \%)$ \\
\hline
\end{tabular}

En base a las ecuaciones indicadas en la Tabla 3 se calcularon los tiempos teóricos de aparición de la primera fisura obtenidos de los diagramas de Tutti. En la Figura 6 se muestran los valores calculados. En las vigas con $C N$ la aparición se produce entre los 8 y 21 días, en cambio con los curados $C L, C M$ y $C E$ se produce un retraso promedio del $215 \%, 180 \%$ y $143 \%$, respectivamente. Con el curado $C L V$, los tiempos son prácticamente similares a los del $C N$. 
Revista ALCONPAT, Volumen 2, Número 2, Mayo - Agosto 2012, Páginas 72 - 81

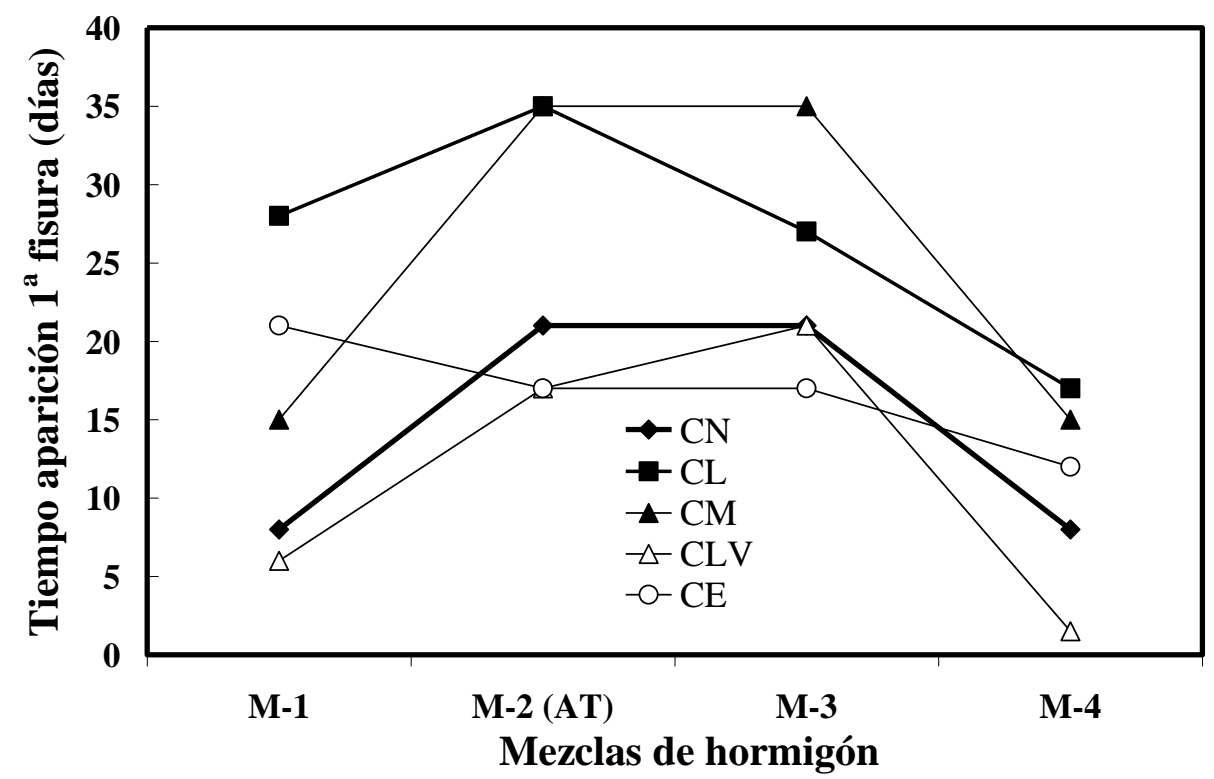

Figura 6. Tiempo teórico de aparición de la primera fisura.

Respecto a los mínimos potenciales de corrosión medidos, las mezclas con $C N$ se mantuvieron entre -500 y $-560 \mathrm{mV}$, y en las restantes vigas en un rango algo mayor, -460 y $-600 \mathrm{mV}$. Las pérdidas de acero de los $50 \mathrm{~cm}$ centrales de las vigas, que incluye la parte superior de los estribos, no muestran diferencias importantes para los distintos curados. Las pérdidas fueron de $30.8 \%$, $28.4 \%, 32.8 \%, 34 \%$ y $35 \%$ para los curados $C N, C L, C M, C E$ y $C L V$, respectivamente.

Influencia del tipo de agregado fino: independientemente del curado, las cuatro mezclas presentan al final del ensayo, valores de áreas de fisuración diferentes, y en la Figura 7 se muestra la tendencia que relaciona estas áreas con la VSC. La línea llena superior corresponde a la tendencia de todos los valores estudiados con diferentes tipos de curados. Los coeficientes de correlación de las rectas de cada curado van disminuyendo a medida que este resulta menos favorable. Las tendencias que se establecen en la figura muestran que cuando se incrementa la succión capilar, es decir que el material es más poroso o sus poros están más interconectados, la presión que ejercen los productos de corrosión en el interior de ellos disminuye, y en consecuencia merma la fisuración o grado de deterioro de las vigas.

Por otra parte, en la Figura 8 se muestra la relación entre el daño de las vigas y el módulo de finura de la arena, para los curados estudiados. Para los $C N, C E, C L$ y $C L V$ a medida que el módulo de finura disminuye, las partículas son más pequeñas, la superficie específica es mayor y el espesor de las interfaces "pasta-agregado fino" es menor. De este modo se obtuvieron áreas de fisuras mayores ante una mayor presión de los productos de hidratación. En cambio, cuando las probetas se curan a temperaturas del orden de $40^{\circ} \mathrm{C}$, el comportamiento es inverso, debido tal vez al proceso de micro-fisuración provocado por un secado más agresivo durante los primeros 7 días. 


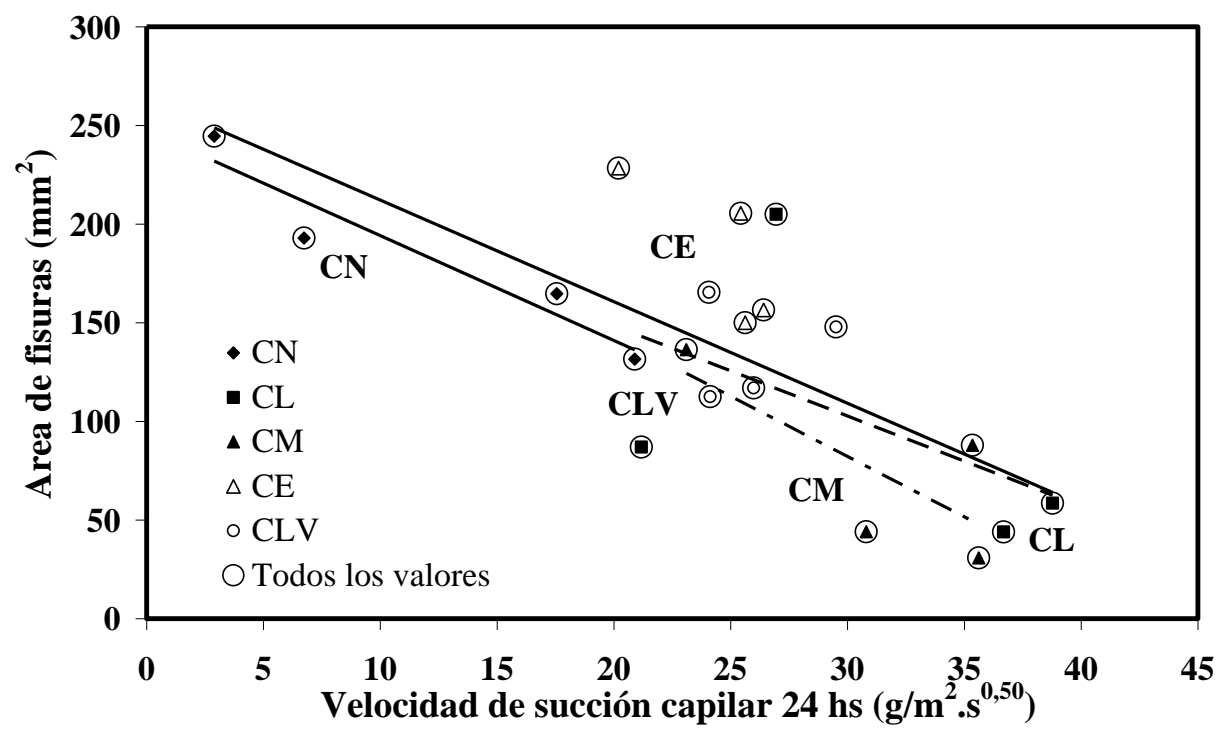

Figura 7. Relación entre el área de fisuras y la velocidad de succión capilar.

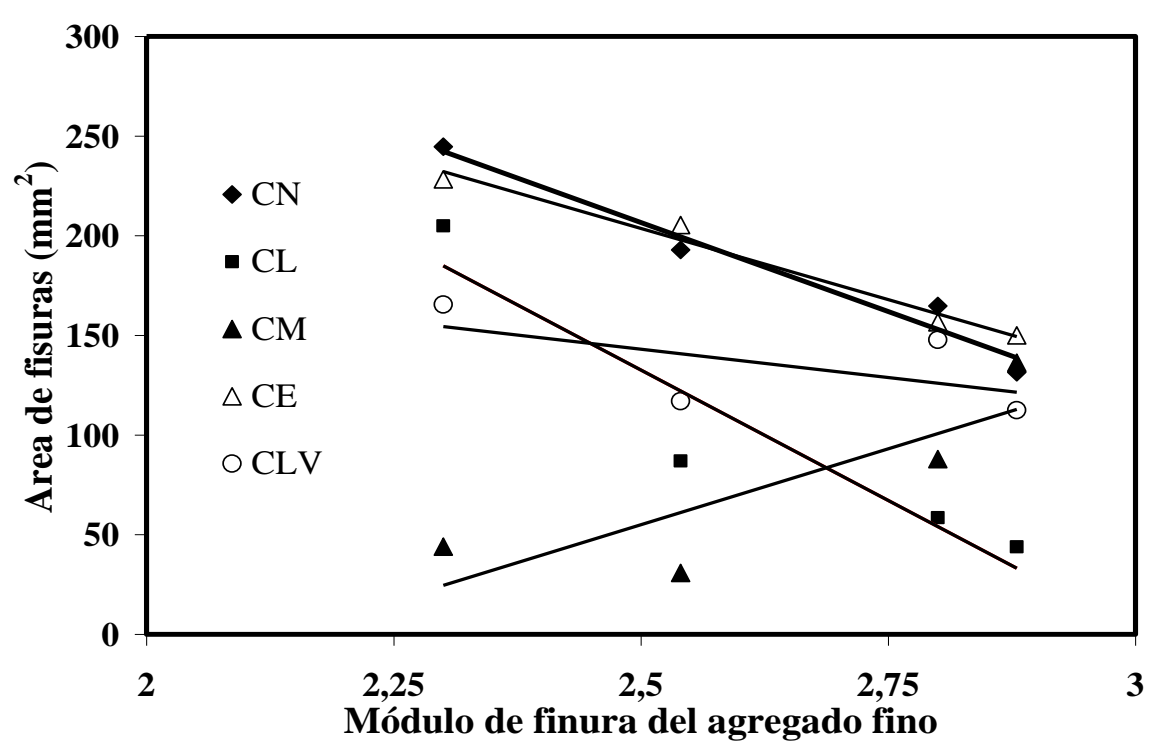

Figura 8. Relación entre el área de fisuras y el módulo de finura de las arenas $\left(\mathrm{R}^{2}=0.98-0.71\right)$.

Finalmente, de modo complementario se indica que el análisis efectuado en base a los anchos máximos de fisuras, para los diferentes curados, presentó tendencias similares al caso de los análisis en base a áreas de fisuras cuando se los compara con el $P M$, la VSC y el módulo de finura de la arena.

\section{CONCLUSIONES}

Los estudios realizados permitieron efectuar las siguientes consideraciones:

- El tipo de curado influye más fuertemente sobre la VSC, que sobre la resistencia del hormigón, dado que el primero es un fenómeno preponderantemente superficial.

- En el caso de la corrosión de las vigas de hormigón armado, el curado defectuoso tiene una importante influencia sobre el tiempo de aparición de la primera fisura, el área de fisuras, y sobre el ancho máximo de fisuras; si bien las fisuras son de menor magnitud, existe una mayor dispersión de resultados entre las mezclas de cada grupo. Además, provoca una mayor porosidad y/o interconexión de poros, que permite disipar la presión de los productos de corrosión, Influencia del curado sobre la evolución de la corrosión en vigas de hormigón armado con... 80 
Revista ALCONPAT, Volumen 2, Número 2, Mayo - Agosto 2012, Páginas 72 - 81

originando un daño menor en el hormigón. Aunque el volumen de acero corroído resultó ser similar, en los cinco grupos de vigas.

- A medida que se incrementa el valor de la VSC hay un menor grado de deterioro que se justifica por la mayor porosidad del material como en la conclusión anterior.

- El deterioro esta ligado también al tipo de agregado fino y, específicamente, a su finura que incide sobre el espesor de la interfaz "pasta-agregado fino", y a la forma y textura de los granos, que determinan la tortuosidad de las interfaces que inciden sobre la ubicación y movimiento de los productos de corrosión.

\section{REFERENCIAS}

Alonso C., Andrade C., Rodríguez J., Diez J. M. (1998), Factors controlling cracking in concrete affected by reinforcement corrosion, Materials and Structures, 31, pp. 435-441.

Aveldaño R., Ortega N. F. (2009), Influence of reinforcement distribution in the corrosive process of reinforced concrete beams, Magazine of Concrete Research, 61, 3, pp. 213-220.

Cabrera O. A., Donza H. A., Traversa L. P. (2006), Influencia de la forma y textura superficial del agregado fino sobre la succión capilar, en Ed. J.D. Sota, $16^{\mathrm{a}}$ Reunión Técnica de la AATH, Mendoza, Argentina, pp. 195-203.

Cabrera O. A., Traversa L. P., Ortega N. F. (2011), Estado fresco de morteros y hormigones con arenas de machaqueo / Effect of crushed sand on mortar and concrete rheology, Materiales de Construcción, 61, 303, pp. 401- 416.

DURAR, (2000), Manual de Inspección, Evaluación y Diagnóstico de Corrosión en Estructuras de Hormigón Armado, Madrid, España, Ed. CYTED, p. 204.

Norma ASTM C-876 (2000), Standard test method for half-cell potential of reinforcing steel in concrete.

Norma IRAM 1546, (1992), Hormigón de cemento pórtland. Método de ensayo de compresión, Buenos Aires, Argentina.

Norma IRAM 50000, (2000), Cemento. Cemento para uso general. Composición, características, evaluación de la conformidad y condiciones de recepción, Buenos Aires, Argentina.

Ortega N. F., Aveldaño R. (2008), Analysis of tensed reinforced concrete beams during the corrosion process, The Open Construction \& Building Technology Journal, Bentham Publishers, 2, pp. 243-250.

Rodríguez J., Ortega L. M., García A. M. (1993), Medida de la velocidad de corrosión de las armaduras en estructuras de hormigón, mediante un equipo desarrollado dentro del proyecto Eureka EU 401, Hormigón y Acero, № 189, pp. 79-91.

Schierloh M. I. (2002), Corrosión de armaduras. Características que debe tener el hormigón para aumentar la protección, Tesis de Maestría, Universidad Nacional del Sur, Argentina, p. 224.

Torres-Acosta A. A. and Sagüés A. (1998), Concrete cover cracking and corrosion expansion of embedded reinforcing steel, in P. Castro, O. Troconis, C. Andrade (Eds.), Rehabilitation of Corrosion Damaged Infrastructure, NACE Internacional, Houston: TX (USA), pp. 215-229.

Traversa L. P. (2001), Corrosión de Armaduras en Atmósferas Rurales, Urbanas, Marinas e Industriales, Capítulo 5, Durabilidad del Hormigón Estructural, La Plata, Argentina, Ed. E. Irassar, AATH, p.306. 